\title{
Remoção de Níquel(II) e Cádmio(II) em N-Lauroil Quitosana em Soluções Aquosas: Cinética e Isotermas
}

\author{
Aline F. Barcelos, Danillo A. Silva, Camilla L. Vieira \& Roberta Signini
}

Quitosana reagiu com cloreto de lauroil para obter o adsorvente N-Lauroil Quitosana(NLQ). O adsorvente foi usado para estudos de adsorção em solução aquosa de íons de níquel(II) e cádmium. Foram realizados estudos de $\mathrm{pH}$, cinéticos e de equilíbrio. $\mathrm{O}$ pH ótimo observado foi de 5,0 e 5,5 para os íons de níquel(II) e cádmium(II), respectivamente. Dos estudos cinéticos, foi observada a cinética de pseudoprimeira ordem. Os dados da adsorção em equilíbrio foram analisados pelos modelos de Langmuir, Freudlinch e Tenkim. A capacidade máxima de adsorção (qmax) foram 20,1 e 49,3 mg $\mathrm{g}^{-1}$ respectivamente para o níquel(II) e cádmio(II).

Palavras-chaves: N-lauroil quitosana; adsorção; ios níquel(II); cádmio(II).

Chitosan was functionalized from lauroyl chloride, obtaining Chitosan-N-Lauroyl adsorbents (NQL). The adsorbent was used to study the adsorption in aqueous nickel(II) and cadmium(II) solution, including $\mathrm{pH}$, kinetics and equilibrium dependence. At optimum pH-value of 5.0 and 5.5 for nickel(II) and cadmium(II) ion in NQL, respectively. The kinetics study demonstrated that the adsorption process proceeded according to the first-second-order model. The equilibrium data were analyzed by Langmuir, Freundlich and Tenkim isotherms models. The maximum adsorption capacities (qmax) were 20.1 and $49.3 \mathrm{mg} \mathrm{g}^{-1}$ for nickel(II) and cadmium(II) ion, respectively.

Palavras-chaves: chitosan-N-lauroyl; adsorption; nickel(II);cadmium(II) ions. 


\section{Introdução}

Aatividade industrial pode gerar condições desfavoráveis ao meio ambiente, contribuído muito para um aumento significativo nas concentrações de íons metálicos em águas, principalmente, os provenientes de metais tóxicos, o que conduz a sérios problemas de acumulação durante os ciclos ecobiológicos. Muitos metais são essenciais para o crescimento de todos os tipos de organismos, desde as bactérias até mesmo o ser humano, mas eles são requeridos em baixas concentrações. Entretanto, os metais tóxicos são extremamente nocivos para uma grande variedade de organismos e representam um grupo de poluentes que requer um tratamento especial, pois não são degradados quimicamente e nem biologicamente, ao contrário são acumulados no ecossistema. Quando absorvidos pelo ser humano, os metais tóxicos se depositam nos tecidos ósseos e gordurosos e deslocam minerais nobres dos ossos e músculos para a circulação, podendo provocar doenças. ${ }^{1-3}$ Apesar dos problemas que os metais podem causar ao ser humano e ao ambiente, não se pode eliminá-los dos processos industriais e, por esta razão, existe a necessidade de estudos em relação à remoção em efluentes de descarte.

São usados para tratar efluentes industriais técnicas de sedimentação, tratamentos eletroquímicos, tratamentos térmicos, tratamentos biológicos, tratamentos físicoquímicos, ${ }^{4}$ eletrodiálise, ${ }^{5}$ osmose reversa e filtração por membrana. ${ }^{6}$ No entanto, estes métodos podem ser ineficazes ou caros, especialmente, quando os íons de metais pesados estão presentes em águas residuais em baixas concentrações. ${ }^{7}$ Portanto, é necessário outros métodos para minimizar as despesas e tornar mais eficiente o tratamento de efluentes que contêm metais em pequenas quantidades. A adsorção é um método mais adequado para ser utilizado em tratamento de efluentes contendo metais, quando esses se encontram em pequenas quantidades. Uma das vantagens desse método em relação aos outros é a baixa geração de resíduos, fácil recuperação de metais e a possibilidade de reutilização do adsorvente em vários ciclos. ${ }^{8}{ }^{89}$ Tem-se uma larga escala de adsorventes à disposição para serem usados para este fim, como, por exemplo, carvão ativado, argilas, sílicas, dentre outros. ${ }^{10}$ Outro tipo de adsorvente, também eficaz e de baixo custo, o que viabiliza o processo, são os chamados bioadsorventes, no qual se podem destacar a quitosana e seus derivados. ${ }^{11-18}$

A capacidade da quitosana em adsorver metais é devido principalmente à presença de grupos aminos(-NH2) que estão presentes em sua estrutura ${ }^{19}$ e esta capacidade varia de acordo com a cristalinidade, afinidade por água, porcentagem de desacetilação e quantidade de grupos amino $^{20}$. A quitosana, apesar de ter grande potencial, possui algumas limitações, como estabilidade mecânica baixa, baixa solubilidade, baixa porosidade, baixa resistência térmica e baixa área superficial. ${ }^{21}$ Portanto, uma modificação em sua cadeia poderá solucionar algumas de suas restrições possibilitando aumento da sua aplicabilidade, por exemplo, para a adsorção de metais.

A modificação da quitosana, geralmente, ocorre de maneira mais fácil quando comparados com outros polissacarídeos, isto é devido os seus grupos funcionais amino e hidroxil serem reativos. ${ }^{22}$ Esses grupos permitem a modificação da quitosana como a acetilação, alquilação, carboximetilação ${ }^{23}$ e formação de bases de Schiff com aldeídos e cetonas. ${ }^{24}$ Assim, neste trabalho, produziu-se um derivado de quitosana, a partir da reação da quitosana com cloreto de lauroila obtendo o N-lauroil quitosana e utilizou este adsorvente para a adsorção de íons metálicos níquel(II) e cádmio(II).

\section{Metodologia}

\section{Síntese e Purificação N-Lauroil Quitosana (NLQ)}

Foram suspensos $10 \mathrm{~g}$ de quitosana em $600 \mathrm{~mL}$ de solução ácido acético $1 \%$ (v/v), a suspensão foi mantida sob agitação mecânica durante 24 horas. Posteriormente, foi ajustado o $\mathrm{pH}$ com hidróxido de sódio $2 \mathrm{~mol} \mathrm{~L}^{-1}$ para atingir pH 6,8-7,0. Em seguida, adicionaram-se lentamente 5,0 mL de cloreto de lauroíla e deixados sob agitação mecânica por 6 horas com rotação de $1250 \mathrm{rpm}$. Posteriormente, foi ajustado novamente o $\mathrm{pH}$ com hidróxido de sódio $2 \mathrm{~mol}$ $\mathrm{L}^{-1}$ para atingir $\mathrm{pH} 6,8-7,0$. Por fim, foi adicionado etanol até a precipitação completa. $\mathrm{O}$ gel expandido (precipitado) 
foi filtrado e lavado abundantemente com acetona e seco à temperatura ambiente.

Para purificação da amostra, foi utilizado o sistema Soxhlet, no qual as amostras permaneceram sob refluxo durante 48 horas em $200 \mathrm{~mL}$ de metanol P.A. Posteriormente, as amostras foram secas à temperatura ambiente e trituradas, por meio do almorafiz e pistilo de porcelana, até atingirem o tamanho $600 \mu \mathrm{m}$ medidos por uma peneira.

\section{ESTUDOS DE ADSORÇÃO}

\section{Efeito do pH na adsorção de íons metálicos em $\mathrm{N}$-lauroil quitosana}

Para avaliar o efeito do $\mathrm{pH}$, foram preparadas suspensões contendo $25 \mathrm{mg}$ do adsorvente NLQ em $25 \mathrm{~mL}$ de solução aquosa do íon metálico com concentração de $100 \mathrm{mg} \mathrm{L}^{-1} \mathrm{em}$ intervalo de $\mathrm{pH}$ de 1,0 a 12,0 . A suspensão foi mantida sob agitação a temperatura $25^{\circ} \mathrm{C}$ pelo período de $2 \mathrm{~h}$. Após, as suspensões foram filtradas e diluídas dez vezes para serem analisadas no espectrofotômetro de absorção atômica AAnalyst 400 da Perkin Elmer. O experimento foi feito em triplicata.

\section{Efeito do tempo de contato na adsorção do íon metálico - Tempo de Equilíbrio e Cinética}

Para que se realizasse esse experimento, foi utilizado um condutivimetro marca GEHAKA com o objetivo de monitorar o tempo, contado em relação à condutividade da amostra. $25 \mathrm{mg}$ de N-lauroil quitosana (NQL) foram adicionados á $25 \mathrm{~mL}$ da solução de íons metálicos em recipiente que possui $100 \mathrm{mgL}^{-1}$ do metal. A solução foi deixada em agitação e à temperatura ambiente e de tempos em tempo foi medida a sua condutividade.

\section{Equilíbrio de adsorção dos íons metálicos em $\mathbf{N}$-Lauroil quitosana}

O estudo de equilíbrio foi conduzido a $25^{\circ} \mathrm{C}$ utilizando $25 \mathrm{mg}$ de NLQ em $25 \mathrm{~mL}$ de solução do íon metálico, sendo que a concentração do íon metálico na solução variou de 20 $\mathrm{mg} \mathrm{L}^{-1}$ a $100 \mathrm{mg} \mathrm{L}^{-1}$. A suspensão foi mantida sob agitação contínua durante o tempo de equilíbrio e $\mathrm{pH}$ igual 5,0 para o caso do íons níquel(II) e 5,5 para os íons cádmio(II). Após a suspensão, foi filtrada e diluída 10 vezes. Em seguida, foram realizadas medidas no espectrofotômetro de absorção. Este experimento foi feito em triplicata.

\section{Resultados e Discussão}

\section{EFEITO DO PH NO PROCESSO DE ADSORÇÃO}

A mudança no $\mathrm{pH}$ afeta o processo de adsorção através da dissociação dos grupos funcionais que estão presentes no material. Na Figura 1, é mostrado o efeito do $\mathrm{pH}$ na adsorção dos íons metálicos de níquel(II) e cádmio(II) em N-Lauroil Quitosana (NLQ). No estudo do efeito do pH na adsorção do íon metálico, variou-se o pH de 1,0 até 12,0. Entretanto, observou-se que para a adsorção dos íons metálicos em $\mathrm{pH} \geq 4,5$ ocorreu a dissolução parcial do adsorvente e em $\mathrm{pH} \geq 7,0$ observou-se precipitação dos íons metálicos na forma de hidróxidos. A dissolução parcial do adsorvente e a precipitação dos íons metálicos pode comprometer o resultado de adsorção, apresentando erros de análises relativamente grandes e, assim, a faixa propriamente estudada de $\mathrm{pH}$ foi de 5,0 a 7,0.

Analisando a Figura 1(a), observa-se que em $\mathrm{pH}$ 5,0 a adsorção de íons de níquel(II) foi em torno de $22 \%$. Ao aumentar o pH do sistema de adsorção dos íons de níquel(II) observa-se que ocorre uma diminuição na adsorção e tem-se a um máximo em pH 7,0. Em pH 7,0 observou uma adsorção de íons de níquel (II) de $25,6 \%$, porém neste $\mathrm{pH}$ ocorre a precipitação dos íons de níquel(II) o que compromete a análise. Fato semelhante é observado no $\mathrm{pH} 7,0$. Desta forma, o $\mathrm{pH}$ ótimo ocorre em 5,0. Na Figura 1(b) que o $\mathrm{pH}$ ao qual teve maior remoção de íons cádmio(II) foi em 5,5, cerca de 32\%, e de maneira semelhante ao observado para os íons de níquel(II) em $\mathrm{pH} \geq 7,0$ ocorre a precipitação dos íons de cádmio(II).

\section{EFEITO DO TEMPO DE CONTATO NA ADSORÇÃO DO ÍON METÁLICO}

\section{Tempo de Equilíbrio}

Foi realizado o estudo do efeito do tempo de contato para os processos de adsorção de íons de níquel(II) e íons de cádmio(II) em N-Lauroil Quitosana, a fim de determinar o tempo de equilíbrio e a cinética envolvida no processo de adsorção. 
O efeito do tempo de contato no processo de adsorção dos íons níquel (II) em N-Lauroil Quitosana pode ser observado na Figura 2(a). Observa-se que a condutividade do sistema diminui bruscamente até próximo de 90 minutos e posteriormente à condutividade diminuiu suavemente $\mathrm{e}$ em torno de 150 minutos e a partir deste momento mantemse constante. Isto sugere que o tempo de equilíbrio dos íons de níquel(II) em N-Lauroil Quitosana ocorre em torno de 150 minutos. Já para o efeito do tempo de contato dos íons cádmio(II) em N-Lauroil Quitosana pode ser observado na Figura 2(b). Neste caso observa-se que a condutividade do sistema diminui bruscamente até próximo de 75 minutos e posteriormente a condutividade diminuiu suavemente e em torno de 180 e depois se mantem constante. Isto sugere que o tempo de equilíbrio dos íons de cádmio(II) em N-Lauroil
Quitosana ocorre em torno de 180 minutos. O tempo de equilíbrio determina o tempo gasto para saturação dos sítios de ligação do adsorvente, no caso o N-Lauroil Quitosana.

A cinética do processo de adsorção foi determinada empregando modelos de pseudoprimeira ordem, ${ }^{25}$ pseudossegunda ordem ${ }^{26}$ e difusão intrapartícula. ${ }^{27}$ Os parâmetros das regressões lineares para os modelos de pseudoprimeira ordem, pseudossegunda ordem e difusão intrapartícula para a adsorção de íons de níquel(II) e de íons de cádmio(II) em N-Lauroil Quitosana estão mostrados na Tabela 1. Observa-se que o modelo cinético que melhor descreve o processo adsortivo, independente do íon metálico, é o modelo o de pseudoprimeira ordem, pois o valor de coeficiente de correlação (R2) foi que mais se aproximou de 1, indicando um bom ajuste.
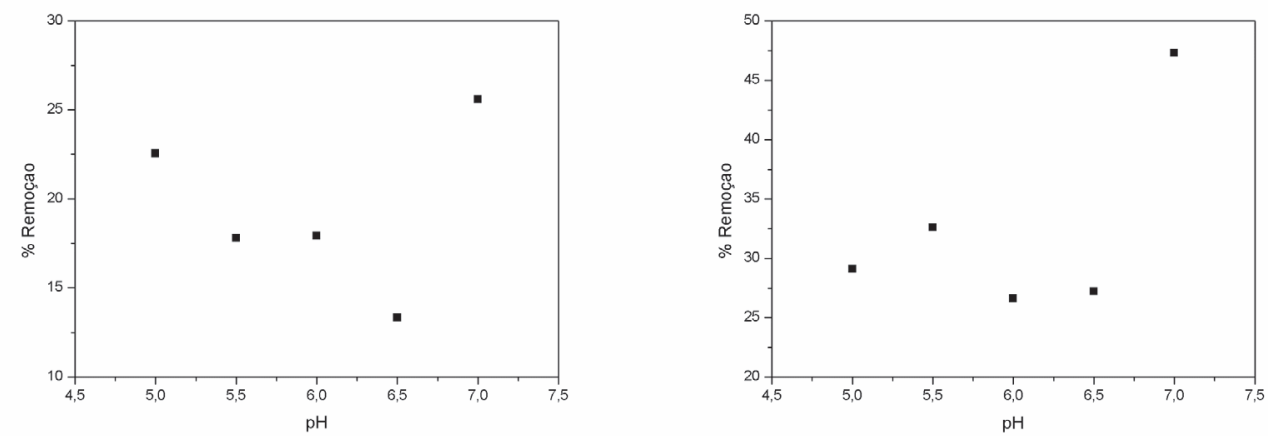

Figura 1. Estudo do efeito do tempo de contato no processo de adsorção de (a) íons de níquel(II) e (b)íons de cádmio(II) em N-Lauroil Quitosana
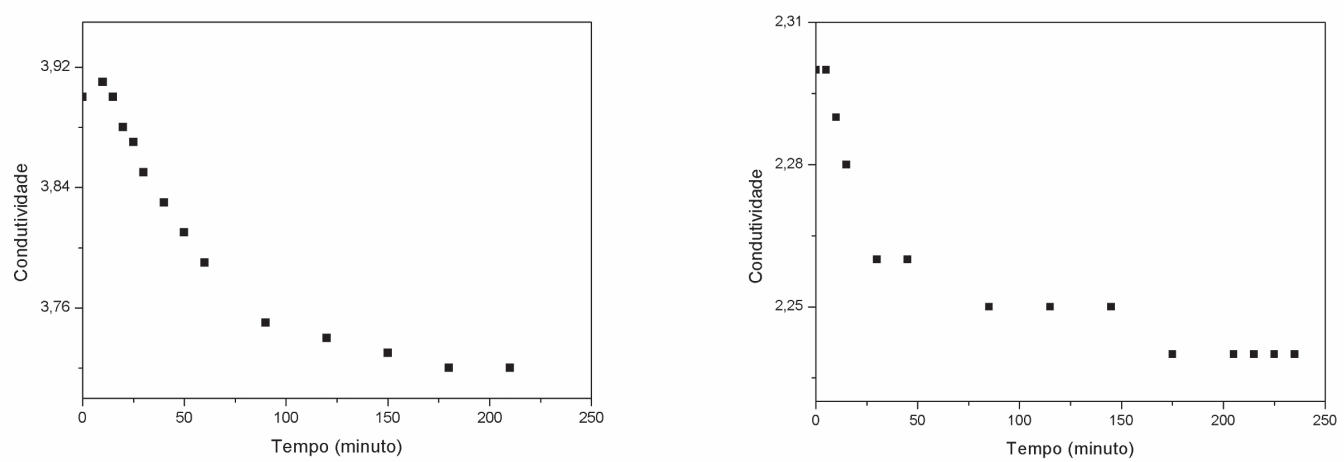

Figura 2. Efeito do tempo de contato no processo de adsorção de (a) Íons de Níquel(II) e (b) Íons de Cádmio(II) em N-Lauroil Quitosana. 
Tabela 1. Resultados cinéticos de adsorção do íons de níquel(II) e íons de cádmio(II) em N-Lauroil Quitosana

\begin{tabular}{|c|c|c|c|c|c|c|}
\hline Íon Metálico & Modelo & $q e\left(m ~ g^{-1}\right)$ & $\mathrm{K} 1\left(\mathrm{~min}^{-1}\right)$ & $\mathrm{K} 2\left(\mathrm{~g} \mathrm{mg}^{-1} \mathrm{~min}^{-1}\right)$ & $\begin{array}{c}\mathrm{Kd}\left(\mathrm{mg} \mathrm{g}^{-1}\right. \\
\left.\min ^{-0,5}\right)\end{array}$ & $\mathbf{R} 2$ \\
\hline \multirow{3}{*}{ Niquel(II) } & Pseudo $1^{\text {a }}$ Ordem & 12,7 & 0,02 & --- & --- & 0,98642 \\
\hline & Pseudo $2^{\mathrm{a}}$ Ordem & 7,3 & --- & 0,01 & --- & 0,84410 \\
\hline & $\begin{array}{c}\text { Difusão } \\
\text { Intrapartícula }\end{array}$ & --- & --- & --- & 0,98 & 0,89381 \\
\hline \multirow{3}{*}{ Cádmio(II) } & Pseudo $1^{\text {a }}$ Ordem & 3,4 & $2,9 \times 10-2$ & --- & --- & 0,88903 \\
\hline & Pseudo $2^{\mathrm{a}}$ Ordem & 3,9 & --- & $1,6 \times 10-2$ & --- & 0,87604 \\
\hline & $\begin{array}{c}\text { Difusão } \\
\text { Intrapartícula }\end{array}$ & --- & --- & --- & $3,9 \times 10^{-1}$ & 0,83387 \\
\hline
\end{tabular}

\section{ESTUDO DE ADSORÇÃO EM EQUILÍBRIO DE ÍONS METÁLICOS}

Nesse estudo, foram empregadas as isotermas de Langmuir, Freundlich e Temkin para se tratarem os dados experimentais. A isoterma de Langmuir prevê que a adsorção (recobrimento) ocorra em apenas uma camada molecular (monocamada), sendo que os sítios de adsorção são considerados uniformes e as moléculas adsorvidas não interagem com os sítios ou moléculas vizinhas. ${ }^{28}$ Assim sendo, a construção da Isoterma de Langmuir (1) se faz a partir de um gráfico Ceq/q versus Ceq. A linearização dos dados, regressão linear, origina uma equação da reta no qual é possível determinar as constantes de Langmuir (KL e qmáx),

$$
\frac{C_{e q}}{q}=\frac{1}{K_{L} \times q_{\max }}+\frac{C_{e q}}{q_{\max }}
$$

Em que: Ceq é a concentração no equilíbrio (mg $\left.\mathrm{L}^{-1}\right)$; q é a quantidade do metal adsorvido ( $\mathrm{mg} \mathrm{g}^{-1}$ ); qmax é a quantidade máxima de metal adsorvido $\left(\mathrm{mg} \mathrm{g}^{-1}\right) ; \mathbf{K}_{\mathrm{L}}$ é a constante de Langmüir $\left(\mathrm{L} \mathrm{mg}^{-1}\right)$.

Pode-se analisar o modelo de Langmuir, a partir das constantes $\mathrm{K}_{\mathrm{L}}$ e qmax, estando essas constantes relacionadas com a energia da adsorção e à capacidade de adsorção máxima. Porém existe ainda outro parâmetro, RL, chamado parâmetro de equilíbrio que é uma constante adimensional que indica se a adsorção é ou não favorável. Para valores entre 0 e 1 , a adsorção é considerada favorável. ${ }^{29} \mathrm{~A}$ constante RL é definida pela equação 2 ,

$$
R_{L}=\frac{1}{\left(1+K_{L} \times C_{0}\right)}
$$

Em que: RL o parâmetro de equilíbrio; KL a constante de Langmuir $\left(\mathrm{L} \mathrm{mg}^{-1}\right)$; $\mathbf{C} \mathbf{0}$ a concentração inicial mais alta do metal $\left(\mathrm{mg} \mathrm{L}^{-1}\right)$.

A isoterma de Freundlich ocorre, geralmente, em multicamadas diferentemente da monocamada exigida pela isoterma de Langmuir, sendo a primeira uma derivação da segunda na qual se modifica a hipótese de que todos os sítios são equivalentes. ${ }^{30} \mathrm{~A}$ construção da Isoterma de Freundich (equação 3) se faz a partir da linearização de um gráfico $\log$ q versus $\log$ Ceq,

$$
\log q=\log K_{F}+\frac{1}{n} \times \log C_{e q}
$$

Em que: Ceq é a concentração no equilíbrio $\left(\mathrm{mg} \mathrm{L}^{-1}\right) ; \mathbf{q}$ é a quantidade do metal adsorvido ( $\mathrm{mg}$ $\left.\mathrm{g}^{-1}\right) ; \mathbf{1} / \mathbf{n}$ é uma constante adimensional relacionada com a intensidade da adsorção, também conhecido como fator de linearidade; $\mathbf{K}_{\mathbf{F}}$ é a constante de Freundlich. 
Temkin 31 propôs um modelo (Equação 4 e 5) em que são considerados os efeitos das interações indiretas entre as moléculas do adsorvato. O modelo de Temkin assume que:

(i) $\mathrm{O}$ calor de adsorção de todas as moléculas diminui linearmente com a cobertura, devido às interações adsorvato-adsorvente;

(ii) A adsorção é caracterizada por uma distribuição uniforme de energias de ligação.

$$
q=B_{T} \cdot \ln K_{T}+B_{T} \ln C_{e q}
$$

Sendo

$$
B_{T}=\frac{R T}{b}
$$

Em que: B é a constante adimensional de Temkin relacionada com o calor de adsorção com o número total de sítios; b é o calor de adsorção $\left(\mathrm{J} \mathrm{mol}^{-1}\right)$; $\mathrm{K}_{\mathrm{T}}$ é a constante de Temkin $\left(\mathrm{L} \mathrm{g}^{-1}\right)$ e $\mathrm{R}$ é constante universal dos gases ideais $\left(8,314 \mathrm{~J} \mathrm{~mol}^{-1} \mathrm{k}^{-1}\right)$. $^{32,33}$

$\mathrm{Na}$ Tabela 2, são mostrados os resultados das isotermas de Langmuir, Freundlich e Temkin para o processo de adsorção de íons de níquel(II) e íons cádmio(II) em N-Lauroil Quitosana.

Baseado no coeficiente de correlação $\left(\mathrm{R}^{2}\right)$, o modelo que melhor descreve o processo de adsorção de íons de níquel(II) e de íons cádmio(II) em N-Lauroil Quitosana foi o de Freundlich, pois o valor do coeficiente de correlação $\left(\mathrm{R}^{2}\right)$ obtidos nesta isoterma estão mais próximo de 1 , sugerindo que o processo de adsorção é em multicamadas.

Tabela 2. Parâmetros obtidos dos modelos das isotermas de adsorção

\begin{tabular}{|c|c|c|c|}
\hline \multirow{2}{*}{ Isoterma } & \multirow{2}{*}{ Parâmetro } & \multicolumn{2}{|c|}{ Adsorvato } \\
\cline { 2 - 4 } & $\mathrm{qmax}(\mathrm{mg} / \mathrm{g})$ & 20,1 & Cádmio(II) \\
\hline \multirow{4}{*}{ Langmuir } & $\mathrm{KL}\left(\mathrm{L} \mathrm{mg}^{-1}\right)$ & 0,04 & 49,3 \\
\cline { 2 - 4 } & $\mathrm{RL}$ & 0,22 & 0,01 \\
\cline { 2 - 4 } & $\mathrm{R} 2$ & 0,950 & 0,50 \\
\cline { 2 - 4 } & $\mathrm{KF}$ & 1,86 & 0,982 \\
\hline \multirow{4}{*}{ Freundlich } & $\mathrm{K}$ & 0,48 & 0,73 \\
\cline { 2 - 4 } & $\mathrm{n}$ & 2,1 & 0,63 \\
\cline { 2 - 4 } & $\mathrm{R} 2$ & 0,965 & 1,6 \\
\cline { 2 - 4 } & $\mathrm{KT}\left(\mathrm{L} \mathrm{g}^{-1}\right)$ & 0,33 & 0,997 \\
\hline \multirow{3}{*}{ Temkin } & $\mathrm{BT}$ & 4,51 & 0,33 \\
\cline { 2 - 4 } & $\mathrm{b}$ & 549,54 & 27,5 \\
\cline { 2 - 4 } & $\mathrm{R} 2$ & 0,943 & 90,1 \\
\cline { 2 - 4 } & & & 0,983 \\
\hline
\end{tabular}

A partir da isoterma de Freundlich para o processo de adsorção de íons de níquel(II) e íons cádmio(II) em N-Lauroil Quitosana, obteve-se um valor de KF (constante de Freundlich) igual a 1,86 e 0,73 , respectivamente. Para valores de $n$ entre 1 e 10, a adsorção é considerada favorável, 34 neste trabalho ambos os valores de $n$ encontrado para o processo de adsorção estão nesta faixa, indicando que o processo é favorável. Fato que também é confirmado pelo parâmetro de equilíbrio, RL, a qual considera o processo de adsorção favorável valores de RL entre 0 e 1 , sendo que neste o trabalho o valor de $\mathrm{RL}$ foi de 0,22 e 0,50 , respectivamente, para o processo de adsorção dos íons de níquel(II) e íons de cádmio(II) em N-Lauroil Quitosana. O valor de RL indica o tipo da 
isoterma, ou seja, se a isoterma é irreversível $(\mathrm{RL}=0)$, favorável $(0<R L<1)$, linear $(R L=1)$ ou desfavorável $(\mathrm{RL}>1) .{ }^{29,35}$ Através da isoterma de Langmuir, observouse que a capacidade máxima absorvida (qmáx) foi de 20,1 mg de íons níquel (II) por grama de N-Lauroil Quitosana e 49,3 $\mathrm{mg} \mathrm{g}^{-1}$ para o processo de adsorção de cádmio(II). Os valores de qmax para os íons metálicos mostra que o adsorvente tem maior afinidade pelo íons de cádmio(II).

$\mathrm{Na}$ isoterma de Temkin, obteve-se um valor de KT (constante de Temkin) de $0,33 \mathrm{~L} \mathrm{~g}^{-1}$ independente do íon metálico considerado já o um valor de BT de foi de 4,51 e 27,5 respectivamente para o processo de adsorção dos íons níquel(II) e cádmio(II) em N-Lauroil Quitosana. Analisando o valor obtido de BT, pode-se perceber que o calor de adsorção das moléculas diminui linearmente com a cobertura da superfície do adsorvente devido às interações existentes. ${ }^{36}$

\section{Considerações Finais}

$\mathrm{O}$ pH ótimo obtido no processo de adsorção no N-Lauroil Quitosana foram 5,0 e 5,5 para os íons níquel(II) e cádmio(II), respectivamente. O tempo de equilíbrio do processo de adsorção foi de 150 e 180 minutos respectivamente para o processo de adsorção de íons de níquel(II) e cádmio(II) em N-Lauroil Quitosana. A cinética apresentada para ambos os processos de adsorção foi de primeira ordem. O valor obtido de n obtido pelo modelo de Freundlich e de RL (parâmetro de equilíbrio) sugere-se que N-Lauroil Quitosana apresenta uma adsorção favorável. O valor de qmax foi de 20,1 e $49,3 \mathrm{mg} \mathrm{g}^{-1}$ respectivamente para o processo de adsorção de íons de níquel(II) e cádmio(II) em N-Lauroil Quitosana. Dos valores de qmax, verifica-se que o N-Lauroil quitosana tem maior afinidade pelos íons de cádmio(II) do que pelo íons de níquel(II).

\section{Agradecimentos}

\section{Referências}

1. Das, S.; Raj, R.; Mangwani, N.; Dash, H.R.; Chakraborty, J. Microb. Biodegrad. Biorem. 2014, 12, 23.

2. Dias, D.L. Metais Pesados. [s.d.]. Disponível em: $<\mathrm{http} / /$ mundoeducacao.bol.uol.com.br/quimica/metais-pesados.htm $>$ acesso em: 26 jan. 2019.

3. Ellingsen, D.G.; Moller, L.B.; Aaseth, J. Copper. Handbook on the Toxicology of Metals. 4 ed., v. 2, p. 765-786, 2015.

4. Akcil, A.; Frust,C.; Ozdemiroglu, S,; Fonte, V.; Beolchini, F. J. Cleaner Prod. 2014, 81, 1.

5. Al-Shannag, M.; Al-Qodah, Z.; Bani-Melhem, K.; Qtaishat, M.R.;Alkasrawi, M. Chem. Eng. J. 2015, 260, 749.

6. Abdullah, A.Z.; Salamatinia, B.; Kamaruddin, A.H. Desalination 2009, 244, 227.

7. Kobya, M.; Demirbas, E.; Senturk, E.; Ince, M. Bioresour. Technol. 2005, 96, 1518 .

8. Benasse, J.C.; Laus, R.; Geremias, R.; Lima, P.L.; Menezes, C.T.B.; Laranjeira, D.; Wilhelm-Filho, D.; Favere, V.T.; Pedrosa, R.C. Arch. Environ. Contam. Toxicol. 2006, 51, 633.

9. Ng, J.C.Y.; Cheung, W.H.; Mckay, G. Chemosphere. 2003, 52, 1021.

10. Costa, C. A.; Schneider, I. A.; Rubio, J. Eng. Sanit. Ambiental. 2000, $5(1 / 2), 19$.

11. Almeida, F.T.R.; Ferreira, B.C.S.; Moreira, A.L.S.L.; Freitas, R.P.; Gil, L.F.; Gurgel, V.A. J. Colloid Interface Sci. 2015, 15, 30421.

12. Borsagli, F.G.L.M.; Mansur, A.A.P.; Chagas, P.; Oliveira, L.C.A.; Mansur, H.S. React. Funct. Polym. 2015, 97, 37.

13. Cui, H.; Chen, J.; Yang, H.; Wang, W.; Liu, Y.; Zou, D.; Liu, W.; Men, G. Chem. Eng. J. 2013, 232, 372.

14. Dragan, E.S.; Cocarta, A.I.; Dinu, M.V. Chem. Eng. J. 2014, 255, 659.

15. Fan, L.; Luo, C.; SUN, M.; LI, X.; QIU, H. Colloids Surf., B. 2013 , $103,523$.

16. Gazargan-Lari, R.; Zafarani, H.R.; Bahrololoom, M.E.; Nemati, A. J. Taiwan Inst. Chem. Eng. 2014, 45, 1642.

17. Huang, J.; Xie, H.; Ye, H.; Xie, T.; Lin, Y.; Gong, J.; Jiang, C.; Wu, Y.; Liu, S.; Cui, Y.; Mao, J.; Mei, L. Carbohydr. Polym. 2016, 138, 301.

18. Shaker, M.A.; Yakout, A.A. Spectrochim. Acta, Part A. 2016, 154, 156.

19. Kyzas, G.Z.; Deliyannt, E.A. Molecules. 2013, 18, 6193.

20. Ying, G.; Xiong, W.; Wang, H.; Sun, Y.; Liu, H. Carbohydr. Polym. 2011, 83, 1787.

21. Crini,G.; Badot, P.-M; Prog. Polym. Sci. 2008, 33, 399.

22. Rinaudo, M. Prog. Polym. Sci. 2006, 31, 603. 


\section{Artigo Geral 7}

23. Shin, Y. Zhu, Y.; Ran, X.; Wang, M.; Su, Y.; Cheng, T. J. Surg. Res. 2006, 133, 185 .

24. Baran, T.; Mentes, A. Int. J. Biol. Macromol. 2015, 79, 542.

25. Ho, Y.; Mckay, G. A. Process Saf. Environ. Prot. 1998, 76, 332.

26. Qiu, H.; LV, L. et al. J. Zhejiang Univ., Sci., A. 2009, 10, 716.

27. Wu, F. C.; Tseng, R. L.; Juang, R. S. Water Res. 2001, 35, 613.

28. Langmuir, I. J. Amer. Chem. Soc, 1916, 30, 2263.

29. Fungaro, D.A.; Da Silva, M. G.. Quim. Nova. 2002, 25(6B), 1081.

30. Atkins P., De Paula J. Físico-química. 8.ed, Editora LTC: Rio de Janeiro, v. 2, 2008

31. Temkin, M. J.; Pyzhev, V. Acta Physiochim USSR. 1940, 12, 217.

32. Monier M.; Ayad, D. M.; Wey, Y.; Sarhan, A. J. Hazard. Mater. 2010, 177,962 .

33. Zhou, L.; Wang, Y.; Liu, Z.; Huang, Q. J. Hazard. Mater, 2009, 161; 995.

34. Yan, H.; Dai, J.; Yang, Z.; Yang, H.; Cheng, R. Chem. Eng. J., 2011, 174(2-3), 586.

35. Sadeghi-Kiakhani, M.; Arami, M.; Gharanjig, K. J. Environ. Chem. Eng. 2013, 1, 406.

36. Zheng, Y.M.; Liu, T.; Jiang, J.; Yang, L.; Fan, Y.; Wee, A.T.; Chen, J.P. J. Colloid Interface Sci. 2011, 356, 741.

\section{Aline F. Barcelos, Danillo A. Silva, Camilla L. Vieira \& Roberta Signini*}

Universidade Estadual de Goiás, Campus de Anápolis de Ciências Exatas e Tecnológicas Henrique Santillo, Caixa Postal 459, CEP 75001-970, Anápolis/GO.

*E-mail: roberta.signini@gmail.com 